Available online at www.iponlinejournal.com

\title{
To study the efficacy of air descemetopexy in the management of various types of descemet membrane detachment based on anterior segment - optical coherence tomography \& to know the role of other gases Perfluoropropane (14\% C3F8) and sulphur hexafluoride (20\% SF6) in failed cases of air descemetopexy
}

\author{
Satya Prakash Singh ${ }^{1}$, Mayank Srivastava ${ }^{2}$, Basant Kumar $\operatorname{Singh}^{3}$, Swarnima Agrahari ${ }^{4}$, Santosh Kumar $^{5 *}$ \\ ${ }^{1}$ Professor and Director, ${ }^{2}$ Professor, ${ }^{3}$ Medical Officer, ${ }^{4}$ Senior Resident, ${ }^{5}$ Associate Professor, Dept. of Ophthalmology, Regional Institute \\ of Ophthalmology at Govt. M. D. Eye Hospital, Allahabad, Uttar Pradesh, India
}

\begin{abstract}
Purpose: To study the efficacy of air descemetopexy in the management of various types of descemet membrane detachment (DMD). Materials and Methods: After ethical committee approval, clinical data of 40 patients during the period of December 2017 to December 2018, who developed DMD after cataract surgery were analysed.

Results: Majority of patients $(n=32,80 \%)$ had severe generalized corneal edema, $n=4$ had moderated corneal edema $\& n=4$ had localized mild corneal edema. Only air was sufficient to reattach DMD in majority of the patients ( $\mathrm{n}=36$, $90 \%$ cases), only 4 patients who had very large dmd failed to attach by air. In these patients repeat descemetopexy by $14 \% \mathrm{C} 3 \mathrm{~F} 8$ gas was done which lead to attachment of descemet membrane with improvement of visual acuity in 3 cases. Complications due to increase intraocular pressure were noted with these gases.

Conclusion: Air descemetopexy is a safe \& efficacious option for DMD repair in most of the cases and it is associated with less postop complications.
\end{abstract}

Keywords: Descemetopexy, Descemet membrane detachment (DMD)

\section{Introduction}

Descemet's membrane detachment (DMD) is a common complication after intraocular surgery. It is the basement membrane of corneal endothelium and it helps keep the endothelial monolayer in place to maintain the corneal clearity. When it gets detached, corneal stroma loses its endothelial pump and aqueous enters the predescemetic space along a tear in the Descemet's membrane, such as that created by a corneal incision or trauma to the Descemet membrane during any ocular surgeries. DMD should be suspected when there is localized or diffuse corneal edema or haziness that persists for over two weeks with a decrease in visual acuity. If the cornea is relatively clear, the presence of DMD can be easily detected by slit-lamp biomicroscopy, but in the presence of severe corneal edema its diagnosis on slit lamp becomes difficult and anterior segment optical coherence tomography (AS-OCT) remains the best imaging tool to diagnose DMD in such cases. Several mechanisms have been proposed that leads to Descemet detachment during cataract surgery. The most common cause is inadvertent insertion of instruments between corneal stroma and Descemet's membrane, as first suggested by 'Scheie' in 1964. In 1928, soon after the advent of slit-lamp biomicroscopy, the first systematic description of DMD in the American literature was made by Bernard Samuels. Although there are reports of spontaneous resolution of DMD but with more successful outcomes from simple pneumodescemetopexy being published, many authors now advocate early treatment, particularly for scrolled, extensive and visually impairing central DMD, to hasten visual rehabilitation and prevent wrinkling, fibrosis and shrinkage of Descemet's membrane that can occur with time and result in poor visual outcome. Considering the significance of DMD discussed earlier and taking into account the lack of any clarity in the existing literature for treatment guidelines i.e. When, How\& Which type of DMD should be treated by Air, C3F8 or SF6 and regarding the efficacy of various substances used as tamponade such as $100 \%$ air, isoexpansile $14 \%$ perfluoropropane $(\mathrm{C} 3 \mathrm{~F} 8)$ and $20 \%$ sulfurhexafluoride (SF6).

We are doing our study to evaluate the effectiveness of air descemetopexy in different types and duration of DMD and why it should be preferred over other gases (14\% C3F8, $20 \%$ SF6)

\section{Aims and Objectives}

1. To study the role of Anterior segment optical coherence tomography in early diagnosis of Descemet membrane detachment.

2. To know the role $\&$ failure rate of air descemetopexy.

3. To know the role of other gases perfluoropropane (14\% C3F8), sulphur hexafluoride (20\% SF6) in failed cases of air descemetopexy.

*Corresponding Author: Santosh Kumar, Dept. of Ophthalmology, Regional Institute of Ophthalmology at govt. M. D. Eye Hospital, Allahabad, Uttar Pradesh, India

Email: drsantosh7373@gmail.com

http://doi.org/10.18231/j.ijooo.2019.032 
4. To observe the anatomical and functional outcomes of air descemetopexy in recent and old cases of DMD

5. To know the factors responsible for failed air descemetopexy.

\section{Materials and Methods}

The proposed study is conducted at Regional Institute of Ophthalmology (M.D. Eye Hospital, Dr. Katju Road, Nakhas Kona, Prayagraj, India) from December 2017 to November 2018, after taking permission from the ethical committee. A written consent from all the patients was taken.

This study included the post cataract surgery patients having descemet's membrane detachment who were treated by itracameral air descemetopexy and other gases such as $14 \%$ C3F8 or $20 \%$ SF6 in failed cases of air descemetopexy.

Examinations \& Investigations done Pre \& postdescemetopexy were After taking the patients demographic data, each of them were examined for Visual Acuity by Snellen's Chart, Slit-Lamp biomicroscopy (to determine the extent \& depth of corneal edema), Anterior segment optical coherence tomography (Carl Zeiss Meditech, Dublin, CA, USA) \& Intraocular pressure recording(non-contact tonometer (Topcon CT-80). Post-descemetopexy follow-up visits were done at $6 \mathrm{hrs}$, day 1,1 week $\& 4$ weeks.

\section{Study design and sample size}

This study was a prospective, cross sectional study.

Total 30 eyes of 30 patients post cataract surgery were included in the study.

\section{Selection of cases}

The inclusion criteria were:

1. The patients with descemet's membrane detachment who underwent cataract surgery.

2. Planar \& Non-planar both types of Descemet's membrane detachment.

3. Both recent and old cases of Descemet membrane detachment.

The exclusion criteria were:

1. The cause of descemet membrane detachment other than cataract surgery.

2. Patients with any pre-existing corneal pathology.

3. Patients with any posterior segment pathologies like optic atrophy, cystoid macular edema, retinal hemorrhage, retinal detachment etc.

\section{Data collection}

The data which were collected from the medical records of the patient included

1. Patients name, age, sex, address and contact number.

2. The eye operated, type of cataract surgery, date of cataract surgery \& place of surgery were noted.

3. Pre-descemetopexy visual acuity, pre and postdescemetopexy intraocular pressure(IOP), location and extent of corneal edema or haziness on slit lamp biomicroscopy, location \& type of DMD whether planar or non planar, with scrolled edges or without scrolled edges and duration between descemetopexy and previous cataract surgery.

4. Other details noted were the type of gas used for anterior chamber injection.

5. Postoperative details at $6 \mathrm{hrs}$, day 1, 1 week, 4 weeks included improvement in corneal edema /haziness seen on slit lamp, status of DM attachment and bestcorrected visual acuity.

\section{Results}

\section{Age and sex distribution}

Total 30 eyes of 30 patients were included in the study. Patients age were distributed in the range of 60-85 years and the mean age of the patients were $72.5 \pm 12.5$ years. Out of total 30 patients, 12 were men and 18 were females.

\section{Types of DMD}

Out of the total 30 patients, 25 patients had Planar type of DMD and only 5 had Non-planar DMD. Out of these 25 patients with planar type of DMD, 20 were without scrolled margins and 5 were with scrolled margins while all 5 nonplanar DMDs were without scrolled margins.

\section{DMD duration}

We divided patients into two groups, first group with the duration of $\mathrm{DMD} \leq 15$ days (time period from the date of cataract surgery till the time of air descemetopexy.) and called it as 'recent' DMDs, second group which included patients with $>15$ days of DMD were called 'old' DMDs. Out of total 30 patients, 19 were recent DMDs and 11 were old.

\section{Visual acuity}

Pre - descemetopexy vision in almost all the patients were poor either FC or HM. Post -descemetopexy BCVA of 22 $(73 \%)$ of the patients out of 30 showed improvement to upto $6 / 6-6 / 18$, while $8(27 \%)$ of the patients showed not much improvement in the final visual outcome and their vision remained between 6/24-HM. Fig. 4 shows the graphical representation of $\mathrm{BCVA}$.

\section{Reattachment rate (\%) of DMD with Air on AS-OCT}

Out of total 30 patients,26 patients $(87 \%)$ i.e 25 planar DMDs, scrolled or without scrolled margins \& 1 non-planar DMD showed reattachment with air descemetopexy on ASOCT in first attempt even at 6hrs of followup and only 4 failed to reattach by air. All these 4 failed cases showed reattachment on repeat descemetopxy by using gas $14 \%$ C3F8.

On applying Fischer exact test for statistical analysis for reattachment rate of DMD by air on AS-OCT, it was found to be statistically significant i.e $\mathrm{p}<0.05$. No post-operative complications were noted with air but patients in whom C3F8 gas was used, almost of them complained of eyeache, 
nausea, vomiting due to raised intraocular pressure and were treated with antiglaucoma drugs.

\section{DMD}

Pre op images

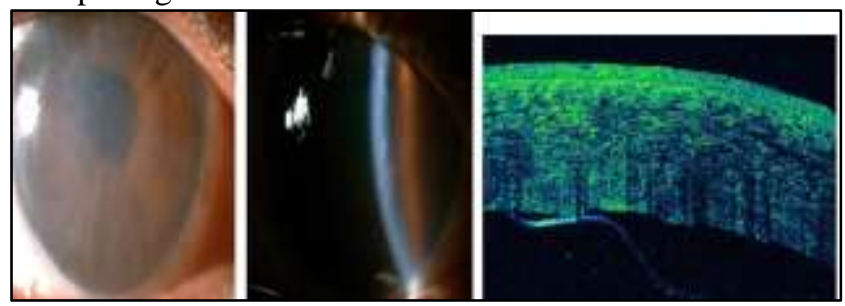

Post op

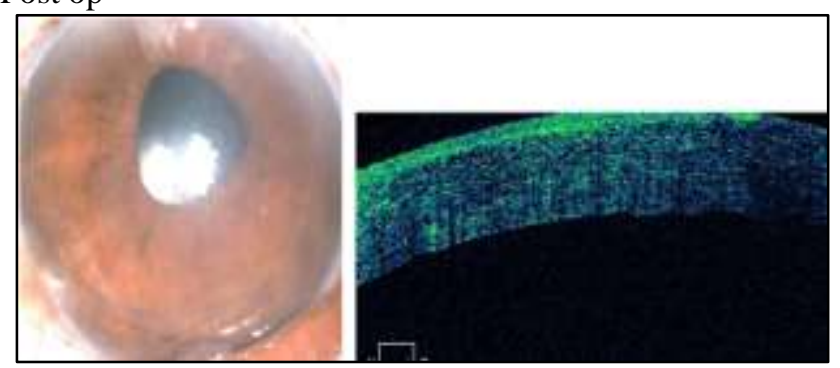

\section{Discussion}

Descemet's membrane detachment (DMD) is a common complication after intraocular surgery specially after cataract surgery. There is a relative paucity in the literature regarding the guidelines for the management of DMD. Some of the questions that still remain unanswered are, whether an intervention is warranted in all cases of DMD, and if required, what duration is ideal, which is the best agent to treat it with, and whether any other factors influence its final outcome.

We studied the role of AS-OCT in early diagnosis of patients with Descemet's membrane detachment and the role of only $100 \%$ air initially in various types of DMD. Other gases such as C3F8 or SF6 were used only if descemet membrane failed to reattach by air in first attempt. We also compared the anatomical \& visual prognosis of air descemetopexy in recent versus old cases of DMD. In the present study, the mean age at the time of presentation was 73 years (range, 60-75 years). There were a total of 17 cases between the age group 60, 70 years, 10 between $71-80$ years and 3 cases more than 80 years. The two studies in India show a relatively younger age group (Rajat Jain53). It may be explained by the fact that cataract occurs early in India.

Majority of the patients were females which similar studies such as Alexander S33, Abraho da Roacha37 and Rajat Jain53. The occurrence of DMD was found more in the female population suggesting delayed surgical intervention. Females are presented to the hospital in the late stages of cataract, when the vision has deteriorated to a large extent or cataract associated complications have occurred. Follow up was also very difficult in female patients as they are relatively more dependent on family members to take them to the hospital.
In our study, all the patients presenting with corneal haze post cataract surgery were examined over slit lamp before going for AS-OCT. Most of the patients showed descemet's membrane detached on slit lamp which was confirmed further on AS-OCT but in the patients with severe corneal edema involving almost entire cornea DMD can be detected only on AS-OCT. Several studies have also shown the use of OCT such as Bryan J. Winn40, Yusen Huang 52, Rajat 53,58, Sonia Kothari62. It is useful to document and follow the postsurgical detachment of DMD and also to determine the site, configuration, and extent of the DMD, thus guiding the treatment.

DMD has been classified by Mackool and Holtz as 'Planar' if separation between Descemet membrane and corneal stroma is $\leq 1 \mathrm{~mm}$ and 'Non-Planar' if separation is $>$ $1 \mathrm{~mm}$ on anterior segment optical coherence tomography (AS-OCT). Types of DMD which we found in our study were mostly Planar. Out of total 30 cases, 25 were planar and only 5 were planar. In 25 planar DMDs, 5 were with scrolled edges. and 20 without scrolled edges.

We observed in our study that the location of DMD whether involving the inferior or superior quadrant of the cornea had no effect on the reattachment rate by air decsemetopexy. While in one of the study done by Namrata Sharma65 etal in which the DMD in superior half of cornea with planar configuration alone were treated by air descemetopexy, and those with scrolled edges were managed by intracameral $14 \% \mathrm{C} 3 \mathrm{~F} 8$ injection. DMDs that were in inferior half of cornea with planar or scrolled edges were managed using $14 \% \mathrm{C} 3 \mathrm{~F} 8$.

In the present study we divided the patients into 2 groups based on duration of DMD. 19 patients who presented within 15 days of cataract surgery, they were classified as 'recent DMD', while those who presented after 15 days of cataract surgery were classified into 'old DMD'. We observed that the duration of DMD had no influence on the anatomical reattachment by air dsecemetopexy, it was similar either with recent or old DMD, but the final visual outcome was influenced by the duration of DMD. There were 8 patients out of total 30 who did not showed improvement in final visual acuity post descemetopexy and out of these 8 patients, 7 were 'old DMD' cases.

There are several reports of spontaneous DMD reattachment by Minkovitz JB8 and Morrison LK9 but delayed repair may be complicated by fibrosis, shrinkage, and wrinkling of DM, which may subsequently prevent reattachment as reported by Mackool RJ14 and Macsai 29.

We treated all 30 cases of Descemet's membrane detachment with intracameral injection of air in the first attempt. Air was used in planar and non-planar both types of DMD, whether with scrolled margins or without scrolled margins. We found that out of 30 cases, 26(87\%) cases showed successful reattachment by air only within $6 \mathrm{hrs}$ post-descemetopexy, but 4 cases failed to attach by air in first attempt and they were scheduled for repeat descemetopexy by $14 \%$ C3F8 gas or $20 \%$ SF6 depending on our senior surgeon's choice. Though these gases lead to 
reattachment of descemet's membrane in failed cases but we observed complications such as vomiting, severe eyeache and raised intraocular pressure in 3 cases out of 4 .

Anterior chamber injection of gas as the primary management strategy has been well described by Makley TA Jr2. It can hasten the absorption of corneal edema and, thus, visual recovery.

BCVA we found that out of the 30 patients $22(73 \% \%)$ patients had final visual acuity between $6 / 6-6 / 18$ whereas 8 (27\%) patients had final visual acuity between 6/24-FC. The BCVA assessment was done after 1 day, 1 week and 1 month of descemetopexy. 8 patients in whom final BCVA doesn't so improvement, 7 of them were 'old DMD' \& only 1 was 'recent DMD'. 5 were Non-planar type \& 3 were of Planar type.

The BCVA was found to be better in eyes who had presented with localised corneal edema and in patients with a good follow up.

4 out of 30 patients required repeat intervention in our study, All these 4 patients had Non-Planar extensive DMDs, these failed to reattach primarly by air only as it does not last longer and gets absorbed within 3 days. These large Non-Planar DMD required gases which lasts longer, so repeat descemetopexy by $14 \% \mathrm{C} 3 \mathrm{~F} 8$ gas was done.

Attachment was seen by $14 \% \mathrm{C} 3 \mathrm{~F} 8$ in all these 4 cases, 1 week post repeat descemetopexy.

\section{Conclusion}

So, we conclude our study by recommending, the use of only Air for almost every type \& duration of DMD for descemetopexy, as the anatomical and visual both outcomes were found significant and isoexpansile gases such as $14 \%$ C3F8 or $20 \%$ SF6 should be used only when air descemetopexy fails to reattach, such as in cases with extensive non-planar DMDs. Post-descemetopexy complications were also high with them as compaired to air, so air is a safe, easy, cheap and effective option for descemetopexy.

\section{Source of Funding}

None.

\section{Conflict of Interest}

None.

\section{References}

1. Jaffe NS. Cataract surgery and its complications. In: Jaffe NS, editor. 4th ed. St. Louis: Mosby, 1984:627-36.

2. Makley TA Jr, Keates RH. Detachment of Descemet's membrane (an early complication of cataract surgery). Ophthalmic Surg 1980;11:189 -91.

3. Samuels B. Detachment of Descemet's membrane. Trans Am Ophthalmol Soc 1928;26:427-37.

4. Scheie HG. Stripping of Descemet's membrane in cataract extraction. Trans Am Ophthalmol Soc 1964;62:140-52.

5. Mulhern M, Barry P, Condon P. A case of Descemet's membrane detachment during phacoemulsification surgery [letter. Br J Ophthalmol 1996;80:185-6.
6. Anderson CJ. Gonioscopy in no-stitch cataract incisions. $J$ Cataract Refract Surg 1993;19:620-1.

7. Monroe WM. Gonioscopy after cataract extraction. South Med $J$ 1971;64:1122- 4 .

8. Minkovitz JB, Schrenk LC, Pepose JS. Spontaneous resolution of an extensive detachment of Descemet's membrane following phacoemulsification. Arch Ophthalmol 1994;112: 551-2.

9. Morrison LK, Talley TW, Waltman SR. Spontaneous detachment of Descemet's membrane. Case report and literature review. Cornea 1989;8:303-5.

10. Bergsma DR Jr, McCaa CS. Extensive detachment of Descemet membrane after holmium laser sclerostomy. Ophthalmol 1996;103:678-80.

11. Macsai MS. Total detachment of Descemet's membrane after small-incision cataract extraction letter. Am J Ophthalmol 1992;114:365- 6 .

12. Al-Mezaine HS. Descemet's membrane detachment after cataract extraction surgery. Int Ophthalmol 2010;30:391-6.

13. Potter J, Zalatimo N. Descemet's membrane detachment after cataract extraction. Optometry 2005;76:720-4.

14. Mackool RJ, Holtz SJ. Descemet membrane detachment. Arch Ophthal 1977;95:459-63

15. Iradier MT, Moreono E. Late spontaneous resolution of a massive detachment of Descemet's membrane after phacoemulsification. JCRS 2002;28:1071-3

16. Hoover DL, Giangiacomo J, B enson RL. Descemet's membrane detachment by sodium hyaluronate. Arch Ophthalmol 1985;103:805-8.

17. Jacob S, Agarwal A. Relaxing descemetotomy relieves stress forces in taut Descemet's membrane detachment. Ocular surgery news.U.S. EDITION October 10, 2010

18. Marcon AS, Rapuano CJ, Jones MR, Laibson PR, Cohen, EJ. Descemet's membrane detachment after cataract surgery management and outcome. Ophthalmol 2002;109:2325-30.

19. Morinelli EN, Najac RD, Speaker MG, Tello C, Liebermann $\mathrm{JM}$, Ritch $\mathrm{R}$ et al. Repair of Descemet's membrane detachment with the assistance of intraoperative ultrasound biomicroscopy. Am J Ophthalmol 1996;121(6):718-20

20. Radhakrishnan S, Goldsmith J, Huang D; et al. Comparison of optical coherence tomography and ultrasound biomicroscopy for detection of narrow anterior chamber angles. Arch Ophthalmol 2005;123(8):1053-9.

21. V Menezo, Y F Choong, N R Hawksworth.Reattachment of extensive Descemet's membrane detachment following uneventful phacoemulsification surgery. Eye 2002;16:786-8.

22. Lee DA, Wilson MR, Yoshizumi MO, Hall M. The ocular effects of gases when injected into the anterior chamber of rabbit eyes. Arch Ophthalmol 1991;109:571-5.

23. P. M. Jacobs, J. M. Twomey, P. K. Leaver. Behaviour of Intraocular References 82 Gases. Eye 1988;2:660-3.

24. Humayun MS, Yeo JH, Koski WS, Michels RG. The rate of sulphur hexafluoride escape from plastic syringe. Arch Ophthalmol 1989;107(6):853-4.

25. Pieramici D, Green WR, Stark WJ. Stripping of Descemet's membrane: a clinicopathologic correlation. Ophthalmic Surg 1994;25(4):226-31.

26. Mark J Walland. Repair of Descemet's membrane detachment after intraocular surgery. J Cataract Refractive Surg 1995;S0886-3350(13)80127-7

27. M Mulhern, P Barry. A case of Descemet's membrane detachment during phacoemulsification surgery. $\mathrm{Br} \mathrm{J}$ Ophthalmol 1996;80:185-8.

28. Muneera A. Mahmood, Klaus D. Teichmann, Karim F. Tomey. Detachment of Descemet's membrane. J Cataract Refractive Surg 1998;24(6):827-33. 
29. Macsai MS, Gainer KM, Chisolm L. Repair of Descemet's membrane detachment with perfluoropropane(C3F8). Cornea 1998;17(2):129-34.

30. C Y W Khng, L W Voon, K T Yeo. Causes and Management of References 83 Descemet's Membrane Detachment Associated with Cataract Surgery - Not Always a Benign Problem. Ann Acad Med Singapore 2001;30:532-5.

31. D. Pahor and B. Gracner. Surgical Repair of Descemet's Membrane Detachment. Coll. Antropol. 25 Suppl. (2001) 1316

32. V Menezo. Reattachment of extensive Descemet's membrane detachment following uneventful phacoemulsification surgery. Eye 2002; 16:786-8.

33. Alexandre S. Marcon, Christopher J. Rapuano, Matthew R. Jones. Descemet's Membrane Detachment after Cataract Surgery: Management and Outcome. Ophthalmol 2002;109:2325-30.

34. Ravinet, E, Tritten, J. J, Roy S. Descemet Membrane Detachment After Nonpenetrating Filtering Surgery. $J$ Glaucoma 2002;11(3):244-52.

35. Ming-Cheng Tai, Feng-Shuen Yieh, Ping-I Chou. Repair of Near Total Descemet's Membrane Detachment with Intracameral Injection of $20 \%$ Sulfur Hexafluoride Gas. J Med Sci 2002;22(5): 231-234

36. In Sik Kim, Jung Chul Shin, Chan Yeong Im. Three Cases of Descemet's Membrane Detachment after Cataract Surgery. Yonsei Med J 2005;46(5):719-23.

37. Abrahao da Rocha Lucena, David da Rocha Lucena, Emília Lucena Macedo. C3F8 use in Descemet detachment after cataract surgery. Arq Bras Oftalmol 2006;69(3):339-43.

38. Ali A, Ahmed T, Ahmed T. Corneal problems during and after phacoemulsification by beginner phacoemulsification surgeon. Pak J Med Sci 2007;23(3):401-4.

39. Briesen S, Roberts H. Repair of Descemet's Membrane Detachment with air. East Afr J Ophthalmol 2007.

40. Bryan J. Winn, Shan C. Lin, Michael R. Hee. Repair of Descemet Membrane Detachments With the Assistance of Anterior Segment Optical Coherence Tomography. Arch Ophthalmol 2008;126(5):730-2.

41. Sevillano C, Viso E. Descemet's membrane detachment as a complication of cataract surgery. Arch Soc Esp Oftalmol 2008;83:549-52

42. Saemah Nuzhat Zafar, Ayesha Khan. Detachment of Descemet's Membrane Following Stromal Hydration in Phacoemulsification Surgery. J Col Physicians Surg Pak 2008;18(3):179-80.

43. Imran Jivraj, Wai-Ching Lam, Varun Chaudhary. Repair of Prolonged Descemet's Membrane Detachments with Intracameral Injection of References 85 Long-Acting Gases. UTMJ 2009;86(2).

44. Akin T, Ayata A, Aykan U, Bilge AH. Intracameral Perfluoropropane (C3F8) Gas in the Repair of Extensive Descemet's Membrane Detachment During Phacoemulsification Surgery. Ophthalmic Surgery, Lasers \& Imaging: the Official Journal of the International Society for Imaging in the Eye 2010:13.

45. Mannan, Rashim, Pruthi, Archna. Descemet Membrane Detachment During Foldable Intraocular Lens Implantation. Eye Contact Lens: Sci Clin Pract 2011;37(2):106-8.

46. Landry H, Aminian A, Hoffart L. Corneal Endothelial Toxicity of Air and SF6. Invest Ophthalmol Vis Sci 2011;52:2279-86

47. Ghaffariyeh A, Honarpisheh N, Chamacham T. SupraDescemet's Fluid Drainage with Simultaneous Air Injection: An Alternative Treatment for Descemet's Membrane Detachment. Middle East Afr J Ophthalmol 2011;18(2):189 91.
48. Perez-Roca F, Legaza SS, Lopez JFR. Treatment of Descemet's membrane detachment after cataract surgery with 20\% SF6. J Emmetropia 2011;2:49-50.

49. Sunita Chaurasia, Muralidhar Ramappa, Prashant Garg. Outcomes of air descemetopexy for Descemet membrane detachment after cataract References 86 surgery. J Cataract Refractive Surg 2012;38(7):1134-9.

50. Aravind Haripriya, David F. Chang, Mascarenhas Reena. Complication rates of phacoemulsification and manual smallincision cataract surgery at Aravind Eye Hospital. J Cataract Refract Surg 2012;38:1360-69

51. Kumar MA, Vaithianathan V. Descemet's membrane detachment managed with perfluro-n-octane liquid. Indian $J$ Ophthalmol 2012;60:71-2.

52. Yusen Huang, Jie Lan, Xinjie Zang. Optical coherence tomography guided intracameral air injection for treatment of extensive Descemet's membrane detachment. Br J Ophthalmol 2012;0:1-2.

53. Rajat Jain, Somasheila I. Murthy, Sayan Basu. Anatomic and Visual Outcomes of Descemetopexy in Post-Cataract Surgery Descemet's Membrane Detachment. Ophthalmol 2013;120:1366-72.

54. Ti, Seng-Ei, Chee, Soon-Phaik, Tan, Donald T. H. Descemet Membrane Detachment After Phacoemulsification Surgery: Risk Factors and Success of Air Bubble Tamponade. Cornea 2013;32(4):454-9.

55. Xixia Ding, Pingjun Chang, Giacomo Savini. Clear Corneal Incision Architecture Imaging after Torsional Phacoemulsification of Hard Nuclear Cataract through a 2.2 $\mathrm{mm}$ and a $3.0 \mathrm{~mm}$ Large Tunnel. J Clin Exp Ophthalmol 2013;4:3.

56. Gujar P, Bhasin P, Bhasin P. Bilateral Descemet's membrane detachment after uneventful phacoemulsification in a case of diabetes mellitus. Indian J Ophthalmol 2013;61:28-9.

57. Asif GH, Kadri WM. Descemet's Membrane Detachment Repair with Sodium Hyaluronate after Phacoemulsification. Pak J Ophthalmol 2013;29(2).

58. Jain R and Mohan N. Outcomes of Repeat Descemetopexy in Post-Cataract Surgery Descemet Membrane Detachment. Am J Ophthalmol 2014;157:571-5.

59. Malhotra R, Garg P, Singh L. Comparison of small incision cataract surgery with conventional extra capsular cataract surgery: an evaluation from resource poor setting in India. Int $J$ Clin Trials 2014;1(1):10-3.

60. Veerendra V.Godbole, Swapnagandha S. Halikar. Air Descemetopexy as a Treatment of Iatrogenic Descemet's Membrane Detachment. IJSR 2014;3(7).

61. Ximenes KF, Silva JV. The role of Descemet's membrane in the pathogeny of corneal edema following anterior segment surgery. Rev Bras Oftalmol 2014;73(5):262-8.

62. Kothari S, Kothari K, Parikh RS. Role of anterior segment optical coherence tomogram in Descemet's membrane detachment. Indian J Ophthalmol 2011;59:303-5.

63. Cagatay HH, Ekinci M, Koban Y. Evaluation of Descemet's Membrane Detachment Using Anterior Segment Optical Coherence Tomography. Turk J Ophthalmol 2014;44:407-9.

64. Su-Ho Lim. Clinical Applications of Anterior Segment Optical Coherence Tomography. J Ophthalmol 2015. Article ID 605729

65. Sharma N, Gupta S, Maharana P. Anterior Segment Optical Coherence Tomography-Guided Management Algorithm for Descemet Membrane Detachment After Intraocular Surgery. Cornea 2015;34:1170-4.

66. Garg J, Mathur U, Acharya MC, Chauhan L. Outcomes of Descemetopexy with Isoexpansile Perfluoropropane after Cataract Surgery. J Ophthalmic Vis Res 2016;11(2):168-73.

67. Annamalai Odayappan, Narayana Shivananda, Seema Ramakrishnan. A retrospective study on the incidence of 
postcataract surgery Descemet's membrane detachment and outcome of air descemetopexy. Br J Ophth

How to cite this article: Singh SP, Srivastava M, Singh BK, Agrahari S, Kumar S. To study the efficacy of air descemetopexy in the management of various types of descemet membrane detachment based on anterior segment optical coherence tomography \& to know the role of other gases Perfluoropropane (14\% C3F8) and sulphur hexafluoride (20\% SF6) in failed cases of air descemetopexy, Int J Ocul Oncol Oculoplasty 2019;5(3):124-9. 